\title{
Quantum Delocalized Interactions
}

\author{
A. J. Paige, ${ }^{1, *}$ Hyukjoon Kwon $\odot,{ }^{1}$ Selwyn Simsek $\odot,{ }^{1}$ Chris N. Self, ${ }^{1}$ Johnnie Gray $\odot,{ }^{1,2}$ and M. S. Kim ${ }^{1}$ \\ ${ }^{1}$ QOLS, Blackett Laboratory, Imperial College London, South Kensington, London, SW7 2AZ, United Kingdom \\ ${ }^{2}$ Division of Chemistry and Chemical Engineering, California Institute of Technology, Pasadena, California 91125, USA
}

(Received 6 May 2020; revised 6 October 2020; accepted 12 November 2020; published 10 December 2020)

\begin{abstract}
Classical mechanics obeys the intuitive logic that a physical event happens at a definite spatial point. Entanglement, however, breaks this logic by enabling interactions without a specific location. In this work we study these delocalized interactions. These are quantum interactions that create less locational information than would be possible classically, as captured by the disturbance induced on some spatial superposition state. We introduce quantum games to capture the effect and demonstrate a direct operational use for quantum concurrence in that it bounds the nonclassical performance gain. We also find a connection with quantum teleportation, and demonstrate the games using an IBM quantum processor.
\end{abstract}

DOI: 10.1103/PhysRevLett.125.240406

Entanglement lies at the heart of the differences between classical and quantum physics. Studying its implications has repeatedly reshaped our understanding of what nature fundamentally allows [1]. In addition to its role in quantum foundations, entanglement is necessary for several types of nonclassical advantage [2-4] and provides the archetypal quantum resource theory [5]. For specific tasks, certain entangled states provide a nonclassical advantage while others do not. Based on this, entanglement can be divided into different levels of hierarchies, such as steering [6,7] and Bell nonlocality [8]. Interestingly, this fundamentally motivated hierarchy has connections to quantum cryptography [9], with corresponding levels of security for entanglement [10], steering [11], and Bell nonlocality [12,13].

A key method for studying particular aspects of entanglement is to consider nonlocal games, where entanglement can provide a nonclassical advantage. The archetypal example is the game constructed from the Clauser-HorneShimony-Holt (CHSH) test [14]. In this CHSH game, Charlie passes two random classical bits $x, y \in\{0,1\}$ to Alice and Bob, respectively. Without communicating to each other, Alice and Bob must select and send back bits $a, b \in\{0,1\}$, respectively, and they win the game if $a \oplus b=x \cdot y$, where $\oplus$ denotes addition modulo 2 . The best classical strategy gives a win probability of 0.75 , but using entangled quantum resources they can win with the maximum probability $\frac{1}{2}(1+1 / \sqrt{2}) \approx 0.85$. Defining and studying games where entanglement provides nonclassical performance has been key to improving our understanding of entanglement [15-27], since these games neatly encapsulate the often counterintuitive consequences for information processing governed by the laws of quantum mechanics.

In this work, we study quantum delocalized interactions, whereby information encoded using nonlocally superposed quantum states, is recorded via local interactions while causing less disturbance than would be classically possible. This indicates that such interactions cannot be said to happen at a single location. This stands in stark contrast to our classical intuition that interactions happen at unique places, we just might not know where. This nonclassical phenomenon has in fact been instrumental in enabling certain quantum protocols $[28,29]$.

In order to characterize delocalized interactions quantitatively, we formulate quantum games and study two particular instances. We establish that the win probabilities of these games are upper bounded in terms of the concurrence for two-qubit states [30,31], and the bounds can be saturated for any pure state and a broad class of mixed states. This provides an operational meaning of the concurrence, which has been a widely studied measure of entanglement but is often viewed as a mathematical device. We find that the capacity for nonclassical teleportation fidelity [32] guarantees the capacity for nonclassical performance in a delocalized interaction game. We also demonstrate the games using an IBM quantum processor, achieving nonclassical performance.

Double slit.-First, we illustrate what we mean by delocalized interactions using the familiar double slit thought experiment. Suppose a game where Charlie $(C)$ either sends a particle through the double slit or does not. Alice $(A)$ standing at one slit together with Bob $(B)$ standing at the other, team up to guess whether $C$ sent the particle or not, without destroying the interference pattern. To win this game, $A$ and $B$ should be able to distinguish between two different states passing through the double slit, namely a vacuum state $|0\rangle$ and a superposition between spatially separated states $\left|\psi_{L}\right\rangle+\left|\psi_{R}\right\rangle$, by locally interacting with the particle. Note these states can also be written as $|00\rangle_{A_{p} B_{p}}$ and $(1 / \sqrt{2})\left(|10\rangle_{A_{p} B_{p}}+|10\rangle_{A_{p} B_{p}}\right)$, where $A_{p}$ and $B_{p}$ are the particle Fock spaces at $A$ and $B$ 's locations. If $A$ and $B$ only 


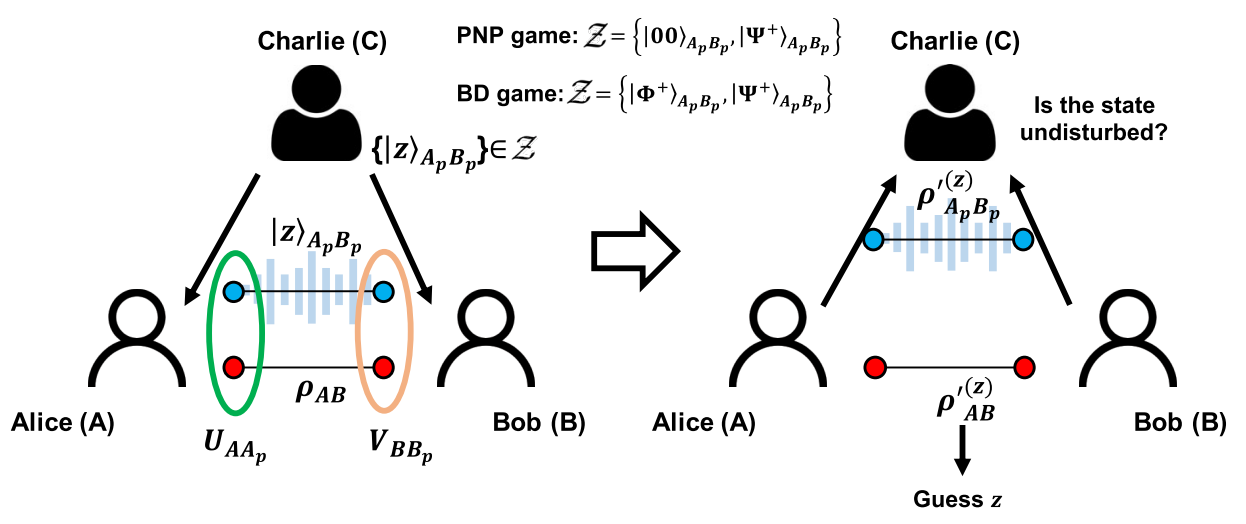

FIG. 1. Schematic illustration of the quantum delocalized interaction games as described in the main text, with $\rho_{A_{p} B_{p}}^{\prime}$ denoting the final state returned to $C$, and $\rho_{A B}^{\prime}{ }^{(z)}$ denoting the final state obtained by $A$ and $B$, which they measure to determine their guess for $z$. The sets of question states used for the PNP game and the BD game are presented at the top.

share classical resources, a perfect record of the existence of the particle is impossible due to the complementarity principle of quantum mechanics. There will be a trade-off, the more information $A$ and $B$ locally record on whether a particle is present, the more they destroy the interference between the different paths by disturbing the superposition state $\left|\psi_{L}\right\rangle+\left|\psi_{R}\right\rangle$ [33]. On the other hand, if $A$ and $B$ share copies of a Bell state, for example, $\left|\Phi^{+}\right\rangle_{A B}=(1 / \sqrt{2})(|00\rangle+|11\rangle)_{A B}$, then they can produce a perfect record of when there were particles without affecting the interference pattern. To do this, $A$ and $B$ set up their local interactions such that the particle flips the local state as $|0\rangle_{A(B)}|1\rangle_{A_{p}\left(B_{p}\right)} \rightarrow|1\rangle_{A(B)}|1\rangle_{A_{p}\left(B_{p}\right)}$ and $\quad|1\rangle_{A(B)}|1\rangle_{A_{p}\left(B_{p}\right)} \rightarrow$ $|0\rangle_{A(B)}|1\rangle_{A_{p}\left(B_{p}\right)}$, while the local states remain the same when the particle is not present. Under this interaction, the resulting joint state evolves as $\left|\Phi^{+}\right\rangle_{A B}\left(\left|\psi_{L}\right\rangle+\left|\psi_{R}\right\rangle\right) \rightarrow$ $(1 / \sqrt{2})(|01\rangle+|10\rangle)_{A B}\left(\left|\psi_{L}\right\rangle+\left|\psi_{R}\right\rangle\right)=\left|\Psi^{+}\right\rangle_{A B}\left(\left|\psi_{L}\right\rangle+\left|\psi_{R}\right\rangle\right)$ when $C$ sent the particle or $\left|\Phi^{+}\right\rangle_{A B}|0\rangle \rightarrow\left|\Phi^{+}\right\rangle_{A B}|0\rangle$ when $C$ does not send the particle. The interference patterns of the particle have not been disturbed and $A$ and $B$ will have a perfect record of the existence of the particle as their shared outcome states $\left|\Phi^{+}\right\rangle_{A B}$ and $\left|\Psi^{+}\right\rangle_{A B}$ are perfectly distinguishable.

As illustrated in the double-slit experiment, entanglement allows us to overcome the trade-off between "information gain via local interaction" and "disturbance in nonlocal superposition" i.e., recording information encoded using nonlocally superposed quantum states, via local interactions while causing less disturbance than would be classically possible. We term this phenomenon delocalized interactions, as the interaction cannot be known to have definitely happened at either $A$ or $B$ 's location, since this would destroy the nonlocal superposition. We proceed to construct a formal quantum game to quantitatively capture the advantage of sharing entanglement between $A$ and $B$ when demonstrating delocalized interactions.
Quantum delocalized-interaction games.-We formulate general quantum delocalized-interaction games as follows (illustrated in Fig. 1):

(1) $C$ prepares a state $|z\rangle_{A_{p} B_{p}}$ selected from some finite set of question states $\mathcal{Z}$ with nonzero probability $P_{z}$, and sends the subsystems $A_{p}$ and $B_{p}$ to $A$ and $B$, respectively.

(2) $A$ and $B$ attempt to record the information $z$ onto their shared state $\rho_{A B}$ via local controlled unitaries $U_{A A_{p}}$ and $V_{B B_{p}}$, then return the subsystems $A_{p}$ and $B_{p}$ to $C$.

(3) $C$ checks whether the returned subsystems $A_{p} B_{p}$ have been disturbed by performing a projective measurement onto the initial state $|z\rangle_{A_{p} B_{p}}$.

(4) $A$ and $B$ perform joint measurements $\Pi_{A B}^{\left(z_{a}\right)}$ to determine their answer $z_{a}$.

(5) $A$ and $B$ win the game if their answer is correct $z_{a}=z$, and $C$ 's projective measurement returns the initial state $|z\rangle_{A_{p} B_{p}}$.

The question states must not be chosen such that $A$ and $B$ cannot distinguish them, and at least one $|z\rangle_{A_{p} B_{p}} \in \mathcal{Z}$ must be entangled. This condition ensures that the games capture the classical trade-off which a quantum delocalized interaction circumvents.

The probability that $A$ and $B$ win the game is given as

$p\left(\rho_{A B}\right)=\sum_{z} P_{z} \operatorname{Tr}\left[\left(\Pi_{A B}^{(z)} \otimes|z\rangle\langle z|\right) W\left(\rho_{A B} \otimes|z\rangle\langle z|\right) W^{\dagger}\right]$,

where $W=U_{A A_{p}} \otimes V_{B B_{p}}$. We shall use the superscript form $p^{m}$ to denote the maximum of this quantity over all choices of measurements $\Pi_{A B}$ and controlled unitaries $U_{A A_{p}}, V_{B B_{p}}$, and we shall use subscripts to distinguish specific instances.

Particle-no-particle game.-The double-slit scenario can now be simplified into an example of a quantum delocalized-interaction game. In this case, $\mathcal{Z}=\{|p\rangle,|n p\rangle\}$ 
with $P_{p}=1 / 2=P_{n p}$ and we take $|p\rangle=(1 / \sqrt{2})\left(|01\rangle_{A_{p} B_{p}}+\right.$ $\left.|10\rangle_{A_{p} B_{p}}\right)$, and $|n p\rangle=|00\rangle_{A_{p} B_{p}}$, which represent the states after passing the double slit depending on whether $C$ sends $(p)$ or does not send $(n p)$ the particle.

We also choose to work with the interaction only happening if the particle exists in the local subsystem, since a unitary in the absence of a particle physically corresponds to free evolution which we can simply factor out. Hence, $U_{A A_{p}}=\mathbb{1}_{A} \otimes|0\rangle_{A_{p}}\left\langle 0\left|+U_{A} \otimes\right| 1\right\rangle_{A_{p}}\langle 1|$ and $V_{B B_{p}}=\mathbb{1}_{B} \otimes|0\rangle_{B_{p}}\left\langle 0\left|+V_{B} \otimes\right| 1\right\rangle_{B_{p}}\langle 1|$. The overall interaction then can be written as

$$
\begin{aligned}
W= & \mathbb{1}_{A B} \otimes|00\rangle_{A_{p} B_{p}}\left\langle 00\left|+U_{A} \otimes \mathbb{1}_{B} \otimes\right| 10\right\rangle_{A_{p} B_{p}}\langle 10| \\
& +\mathbb{1}_{A} \otimes V_{B} \otimes|01\rangle_{A_{p} B_{p}}\langle 01| \\
& +U_{A} \otimes V_{B} \otimes|11\rangle_{A_{p} B_{p}}\langle 11| .
\end{aligned}
$$

We refer to this game as the particle-no-particle (PNP) game and we find that the maximum obtainable win probability for a pure two-qubit state is given as

$$
p_{\mathrm{PNP}}^{m}\left(|\psi\rangle_{A B}\right)=\frac{3}{4}+\frac{1}{4} C\left(|\psi\rangle_{A B}\right),
$$

where $C\left(|\psi\rangle_{A B}\right)=2 \sqrt{\lambda_{0} \lambda_{1}}$ with $\lambda_{i}$ denoting the Schmidt coefficients, is the well-known concurrence entanglement monotone [30,31] (proof in Ref. [34]), which is zero for all separable states, giving the classical bound as $\frac{3}{4}$. We can therefore view the game as providing a direct operational meaning of pure state concurrence.

This result has interesting implications; for instance, one might have thought that $A$ and $B$ would be helped by allowing a preprocessing step where they have temporary access to all the states they will use, and can apply entanglement distillation. However, using the concurrence result we can show that this would not increase their win probability. Consider $A$ and $B$ granted preprocessing access to $N$ copies of the qubit state $|\psi\rangle$, from which they distill $m$ copies of the maximally entangled state and $N-m$ pure separable states. Then when the game starts they use these new states one by one, and win $m$ cases with probability 1 and $N-m$ cases with the maximum classical win probability $\frac{3}{4}$. It is known that in the asymptotic limit of large $N$ we have $m=N E(|\psi\rangle)$, where $E(|\psi\rangle)$ is the entanglement entropy [39]. This means the win probability for the outlined distillation strategy will be bounded by $\quad(1 / N)\left[N E(|\psi\rangle)+\frac{3}{4}(N-N E(|\psi\rangle)]=\frac{3}{4}+\frac{1}{4} E(|\psi\rangle)\right.$. However by using the original states they would obtain $\frac{3}{4}+\frac{1}{4} C(|\psi\rangle)$, and it is known that $C(|\psi\rangle) \geq E(|\psi\rangle)$. Therefore the distillation does not provide improvement.

To generalize Eq. (3) to mixed states we use the fact that the maximum win probability is a convex function $p^{m}\left(\sum_{i} r_{i} \rho^{(i)}\right) \leq \sum_{i} r_{i} p^{m}\left(\rho^{(i)}\right)$, which can be intuitively understood as follows. Consider $A$ and $B$ being either given copies of a known state $\sum_{i} r_{i} \rho^{(i)}$, or given labeled copies of known states $\rho^{(i)}$ where the number of each is in proportion to $r_{i}$. From the second case they can reproduce the first case by simply ignoring the labels, therefore in the second case they must be able to obtain at least as high a win probability as in the first case, hence the convexity result. Using this, combined with the fact that $C\left(\rho_{A B}\right)=\inf \sum_{i} q_{i} C\left(\left|\psi_{i}\right\rangle_{A B}\right)$, we can extend Eq. (3) to a bound for mixed states, giving

$$
p_{\mathrm{PNP}}^{m}\left(\rho_{A B}\right) \leq \frac{3}{4}+\frac{C\left(\rho_{A B}\right)}{4} .
$$

Since the concurrence has an analytic closed form, we can now easily calculate a bound on the win probability gain for any two-qubit state.

From this we can also view the game as providing a direct operational meaning of concurrence for mixed states that saturate the bound. It is therefore natural to ask whether the bound can be tight for mixed states. The answer is yes, as we found that it saturates for mixtures of two Bell states [34]. However, this is not true for all mixed states. An informative example is given by Werner-like states [40] $\rho_{A B}=a\left|\psi^{k}\right\rangle_{A B}\left\langle\psi^{k}\right|+(1-a / 4) \mathbb{1}_{A B}$, where $0 \leq a \leq 1$ and $\left|\psi^{k}\right\rangle$ is chosen as one of the four Bell states. We shall show that this state does not saturate the concurrence bound.

To understand and prove this behavior we note that the mixedness of a state can degrade its record quality. Consider the extreme example of the maximally mixed state $\mathbb{1}_{A B} / n$. It is clear that if $A$ and $B$ try to unitarily encode the presence of a particle in this state then they will not gain information. This inability of the state to acquire information is what we intuitively mean when we say it has bad record quality. We capture the general effect via the bound

$$
p_{\mathrm{PNP}}^{m}\left(\rho_{A B}\right) \leq \frac{1}{2}+\frac{1}{2} T_{c}\left(\lambda^{\uparrow}, \lambda^{\downarrow}\right),
$$

where we denote the classical trace distance $T_{c}(p, q)=$ $\frac{1}{2} \sum_{i}\left|p_{i}-q_{i}\right|$ for probability vectors $p, q$ defined over the same index set, and $\lambda^{\uparrow}$ is the vector of eigenvalues of $\rho$ arranged in ascending order and including any zero values. For the $\mathbb{1}_{A B} / n$ example, we see that the win probability cannot exceed $\frac{1}{2}$, i.e., the best they can do is just guess. The proof of the bound proceeds via the lemma $T(\rho, \sigma) \leq T_{c}\left(\lambda^{\uparrow}, \mu^{\downarrow}\right)$, where $\mu^{\downarrow}$ is the vector of eigenvalues of $\sigma$ in descending order (see Ref. [34] for details).

Returning to the Werner-like states, we find that this record quality bound can be saturated. This can be demonstrated with $U_{A}=X_{A}=|0\rangle_{A}\langle 1|+| 1\rangle_{A}\langle 0|$, and $V_{B}= \pm X_{B}$, where the sign is chosen to match the sign of $\left\langle\psi^{k}\left|X_{A} X_{B}\right| \psi^{k}\right\rangle$. This gives $p_{\mathrm{PNP}}=\frac{1}{2}(1+a)$, which exactly saturates the record bound and is therefore an optimal tactic. This record bound is below the concurrence bound for all $a<1$, and therefore the Werner-like states cannot in general saturate the concurrence bound. 
Since the Werner-like state is entangled for $a>\frac{1}{3}$, these results indicate that entanglement is not sufficient to observe nonclassical advantage in the PNP quantum game. Additionally we note that the capacity for Bell nonlocality is not necessary for a state to demonstrate nonclassical performance, since there is a local model for projective measurements for $a \lesssim 0.66$ [41]. We note that this appears to hold even if we allow $A$ and $B$ to use additional pure classical states (see [34] for results of numerics using qutip $[42,43])$.

Bell distinguishing game.-We now study a modified game that indicates an even stronger connection with concurrence. In the PNP game considered above, the no particle state $|n p\rangle=|00\rangle_{A_{p} B_{p}}$, has no spatial superposition which can be damaged by the local measurements. To move away from this, we can consider replacing $|00\rangle_{A_{p} B_{p}}$, with the Bell state $\left|\Phi^{+}\right\rangle_{A_{p} B_{p}}=(1 / \sqrt{2})\left(|00\rangle_{A_{p} B_{p}}+|11\rangle_{A_{p} B_{p}}\right)$. So Alice and Bob are now tasked with distinguishing two Bell states $\left|\Psi^{+}\right\rangle$and $\left|\Phi^{+}\right\rangle$while trying to return them undamaged. We shall refer to this as the Bell-distinguishing (BD) game. It is noteworthy that this task can be viewed as detecting local bit-flip errors, where in contrast to a conventional syndrome measurement [44] one is using two ancilla modes, each of which can only interact with its local part of the system.

For two-qubit states we again find that the concurrence quantifies the maximum obtainable win probability [34], via

$$
p_{\mathrm{BD}}^{m}\left(|\psi\rangle_{A B}\right)=\frac{1}{2}+\frac{1}{2} C\left(|\psi\rangle_{A B}\right),
$$

and thus we have the general bound

$$
p_{\mathrm{BD}}^{m}\left(\rho_{A B}\right) \leq \frac{1}{2}+\frac{1}{2} C\left(\rho_{A B}\right) .
$$

Unlike for the PNP game, Werner-like states can saturate the concurrence bound, and in fact we find that the well studied Bell diagonal states [45-50] can all saturate the bound. In order to prove this, we note that there exists a tactic with win probability at least equal to the fully entangled fraction (singlet fraction) $\mathcal{F}(\rho)=\max _{\psi}\langle\psi|\rho| \psi\rangle$, where the maximum is taken over all maximally entangled states of the system. $A$ and $B$ can achieve this by adopting the optimal tactic for the maximally entangled pure state $\left|\psi^{*}\right\rangle=\operatorname{argmax}_{\psi}\langle\psi|\rho| \psi\rangle$. Since all entangled Bell diagonal states have concurrence $C(\rho)=2 \mathcal{F}(\rho)-1$, so the tactic outlined above leads to $p_{\mathrm{BD}}(\rho)=\frac{1}{2}+\frac{1}{2} C(\rho)$ thus saturating the bound.

The above outlined tactic also produces an interesting corollary regarding quantum teleportation [32], namely that all entangled two-qubit states capable of nonclassical teleportation fidelity are also capable of nonclassical performance in the $\mathrm{BD}$ game, since it is known that a two-qubit state can achieve nonclassical teleportation fidelity if and only if $\mathcal{F}>\frac{1}{2}$ [51,52]. It would be an interesting open question to study whether the converse statement is true. We conjecture that this might be the case by numerically verifying that examples of entangled twoqubit states with $\mathcal{F}<\frac{1}{2}$ [53] do not show nonclassical BD performance.

IBM machine demonstration. - We tested the delocalized-interaction games using the IBM superconducting quantum processor Paris. We implemented both the BD and PNP games by initially sharing a Bell state between $A$ and $B$ and by employing controlled bit flips as local interaction unitary operators (for details see Ref. [34]). A key simplifying aspect of this approach is that $A$ and $B$ do not have to perform a joint measurement at the end to determine their answer. The two possible states are $(1 / \sqrt{2})\left(|00\rangle_{A B}+|11\rangle_{A B}\right)$ and $(1 / \sqrt{2})\left(|01\rangle_{A B}+|10\rangle_{A B}\right)$, so they can simply measure in the local $Z$ basis and base their answer on the joint parity of their results. Therefore, they only require local operations and classical communication rather than joint measurements.

The results for the BD game are illustrated in Fig. 2, where alongside the results for the entangled initial state, we include results for the separable initial state $|00\rangle$ for comparison. The entangled win probability achieved was 0.71 , which is far from the ideal but violates the classical limit of 0.5 . This demonstrates a usable concurrence of 0.42 and thus a convincing delocalized interaction. For the standard PNP game we could not demonstrate nonclassical performance, but altering the game by increasing the probability a particle is sent $P_{p}$, we were able to establish nonclassical performance, although we cannot currently relate this violation directly to an entanglement measure (details in Ref. [34]).

Note that this is not an ideal demonstration of delocalized interactions. Imperfections in the device's behavior

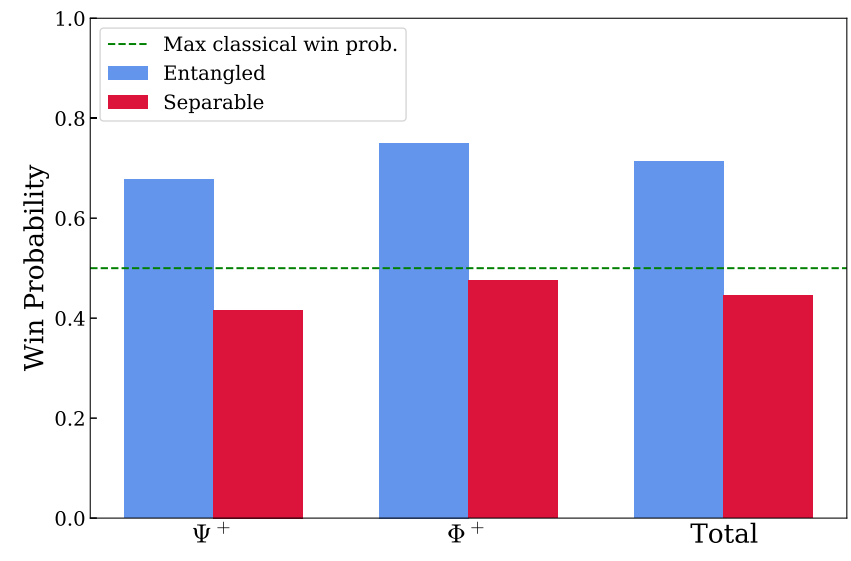

FIG. 2. Plot of results for the BD game, calculated from Paris device measurements. The total win probability is calculated for equal probability of sending either state. The blue bars are for an entangled initial resource state, the red for separable, and the green line is the maximum classical win probability. 
could in principle be used to account for the nonclassical result. Delocalized interactions are subject to the usual loopholes that plague demonstrations of nonlocal effects [54]. Potentially these could be addressed by future experiments with photonic qubits [55].

Conclusions. - In this work we studied the concept of delocalized interactions. Information encoded using nonlocally superposed quantum states is recorded via local interactions while disturbing the superposition less than would be classically possible. This phenomenon has interesting foundational implications regarding events not requiring unique locations and has also been a key component for certain quantum protocols [28,29]. In order to systematically study this quantum effect, we introduced and investigated quantum games for which nonclassical performance demonstrates delocalized interactions. This enabled us to prove a direct operational use of concurrence in bounding the nonclassical win probabilities, and a connection with quantum teleportation. Our work can spur further research building from the tools and ideas introduced here, such as generalizing to higher dimensions or multipartite settings, and establishing the exact nature of the connection with quantum teleportation. Finally, the delocalized-interaction games were demonstrated on an IBM superconducting quantum processor, finding nonclassical performance.

We acknowledge insightful discussions with Benjamin Yadin, David Jennings, Adam Callison, and Thomas Hebdige. A. P. and S.S. are funded by the EPSRC Centre for Doctoral Training in Controlled Quantum Dynamics. We thank the Royal Society, the KIST Open Lab programme, the QuantERA ERA-NET through EP/ R044082/1, and the Samsung GRP grant for financial support.

*a.paige16@imperial.ac.uk

[1] R. Horodecki, P. Horodecki, M. Horodecki, and K. Horodecki, Rev. Mod. Phys. 81, 865 (2009).

[2] R. Jozsa and N. Linden, Proc. R. Soc. Ser. A 459, 2011 (2003).

[3] G. Vidal, Phys. Rev. Lett. 91, 147902 (2003).

[4] V. Giovannetti, S. Lloyd, and L. Maccone, Phys. Rev. Lett. 96, 010401 (2006).

[5] E. Chitambar and G. Gour, Rev. Mod. Phys. 91, 025001 (2019).

[6] E. Schrödinger, Math. Proc. Cambridge Philos. Soc. 31, 555 (1935).

[7] H. M. Wiseman, S. J. Jones, and A. C. Doherty, Phys. Rev. Lett. 98, 140402 (2007).

[8] J. S. Bell, Phys. Phys. Fiz. 1, 195 (1964).

[9] N. Gisin, G. Ribordy, W. Tittel, and H. Zbinden, Rev. Mod. Phys. 74, 145 (2002).

[10] M. Curty, M. Lewenstein, and N. Lütkenhaus, Phys. Rev. Lett. 92, 217903 (2004).
[11] C. Branciard, E. G. Cavalcanti, S. P. Walborn, V. Scarani, and H. M. Wiseman, Phys. Rev. A 85, 010301 (R) (2012).

[12] D. Mayers and A. Yao, in Proceedings of the 39th Annual Symposium on Foundations of Computer Science (Cat. No. 98CB36280) (IEEE, New York, 1998), pp. 503-509.

[13] A. Acín, N. Brunner, N. Gisin, S. Massar, S. Pironio, and V. Scarani, Phys. Rev. Lett. 98, 230501 (2007).

[14] J. F. Clauser, M. A. Horne, A. Shimony, and R. A. Holt, Phys. Rev. Lett. 23, 880 (1969).

[15] R. Cleve, P. Hoyer, B. Toner, and J. Watrous, in Proceedings of the 19th IEEE Annual Conference on Computational Complexity, 2004 (IEEE, New York, 2004), pp. 236-249.

[16] F. Buscemi, Phys. Rev. Lett. 108, 200401 (2012).

[17] C. Branciard, D. Rosset, Y.-C. Liang, and N. Gisin, Phys. Rev. Lett. 110, 060405 (2013).

[18] O. Regev and T. Vidick, ACM Trans. Comput. Theory 7, 15 (2015).

[19] T. Fritz, Rev. Math. Phys. 24, 1250012 (2012).

[20] V. Russo and J. Watrous, arXiv:1709.01837.

[21] N. Johnston, R. Mittal, V. Russo, and J. Watrous, Proc. R. Soc. A 472, 20160003 (2016).

[22] A. Molina and J. Watrous, Proc. R. Soc. A 468, 2614 (2012).

[23] I. Dinur and D. Steurer, in Proceedings of the Forty-Sixth Annual ACM Symposium on Theory of Computing (Association for Computing Machinery, New York, 2014), pp. 624-633.

[24] T. Cooney, M. Junge, C. Palazuelos, and D. Pérez-García, Comput. Complex. 24, 133 (2015).

[25] A. Tavakoli, A. A. Abbott, M.-O. Renou, N. Gisin, and N. Brunner, Phys. Rev. A 98, 052333 (2018).

[26] F. S. Khan, N. Solmeyer, R. Balu, and T. S. Humble, Quantum Inf. Process. 17, 309 (2018).

[27] A. J. Bennet, D. A. Evans, D. J. Saunders, C. Branciard, E. G. Cavalcanti, H. M. Wiseman, and G. J. Pryde, Phys. Rev. X 2, 031003 (2012).

[28] A. Brodutch and E. Cohen, Phys. Rev. Lett. 116, 070404 (2016).

[29] A. Paige, B. Yadin, and M. Kim, Quantum 3, 178 (2019).

[30] S. Hill and W. K. Wootters, Phys. Rev. Lett. 78, 5022 (1997).

[31] W. K. Wootters, Phys. Rev. Lett. 80, 2245 (1998).

[32] C. H. Bennett, G. Brassard, C. Crépeau, R. Jozsa, A. Peres, and W. K. Wootters, Phys. Rev. Lett. 70, 1895 (1993).

[33] B.-G. Englert, Phys. Rev. Lett. 77, 2154 (1996).

[34] See Supplemental Material at http://link.aps.org/ supplemental/10.1103/PhysRevLett.125.240406 for technical proofs and discussions, which includes Refs. [35-38].

[35] G. Vidal and R.F. Werner, Phys. Rev. A 65, 032314 (2002).

[36] M. A. Nielsen and I. Chuang, Am. J. Phys. 70, 558 (2002).

[37] E. M. Rains, Phys. Rev. A 60, 179 (1999).

[38] M. Kjaergaard, M. E. Schwartz, J. Braumüller, P. Krantz, J. I.-J. Wang, S. Gustavsson, and W. D. Oliver, Annu. Rev. Condens. Matter Phys. 11, 369 (2020).

[39] C. H. Bennett, H. J. Bernstein, S. Popescu, and B. Schumacher, Phys. Rev. A 53, 2046 (1996).

[40] R. F. Werner, Phys. Rev. A 40, 4277 (1989). 
[41] A. Acín, N. Gisin, and B. Toner, Phys. Rev. A 73, 062105 (2006).

[42] J. R. Johansson, P. D. Nation, and F. Nori, Comput. Phys. Commun. 183, 1760 (2012).

[43] J. R. Johansson, P. D. Nation, and F. Nori, Comput. Phys. Commun. 184, 1234 (2013).

[44] J. Roffe, Contemp. Phys. 60, 226 (2019).

[45] M. D. Lang and C. M. Caves, Phys. Rev. Lett. 105, 150501 (2010).

[46] Q. Quan, H. Zhu, S.-Y. Liu, S.-M. Fei, H. Fan, and W.-L. Yang, Sci. Rep. 6, 1 (2016).

[47] A. Kay, Phys. Rev. Lett. 109, 080503 (2012).

[48] A. Kent, N. Linden, and S. Massar, Phys. Rev. Lett. 83, 2656 (1999).
[49] L.-X. Cen, N.-J. Wu, F.-H. Yang, and J.-H. An, Phys. Rev. A 65, 052318 (2002).

[50] B.-C. Ren, H.-R. Wei, and F.-G. Deng, Quantum Inf. Process. 13, 1175 (2014).

[51] M. Horodecki, P. Horodecki, and R. Horodecki, Phys. Rev. A 60, 1888 (1999).

[52] P. Badziag, M. Horodecki, P. Horodecki, and R. Horodecki, Phys. Rev. A 62, 012311 (2000).

[53] M. Horodecki, P. Horodecki, and R. Horodecki, in Quantum Information (Springer, New York, 2001), pp. 151-195.

[54] J.-Å. Larsson, J. Phys. A 47, 424003 (2014).

[55] P. Kok, W. J. Munro, K. Nemoto, T. C. Ralph, J.P. Dowling, and G. J. Milburn, Rev. Mod. Phys. 79, 135 (2007). 